\title{
GOCI Data Processing and Ocean Applications
}

\author{
Joo-Hyung Ryu ${ }^{1}$ and Joji Ishizaka ${ }^{2}$ \\ Guest editors of Special Issue \\ ${ }^{1}$ Korea Ocean Satellite Center, KIOST, Ansan P.O.Box 29, Seoul 425-600, Korea \\ ${ }^{2}$ Hydrospheric Atmospheric Research Center, Nagoya University, Aichi 464-8601, Japan
}

(c) KSO, KIOST and Springer 2012

GOCI, the world's first geostationary ocean color sensor, has been developed with the purpose of monitoring the marine environment and providing a quick and timely warning of marine dangers. It takes eight times a day the images of the Northeast Asian region while the polar orbiters have only a 2-3 day revisit occasion. This unprecedented capability allows a breakthrough into a new field of ocean color observation. The hourly shot images of the GOCI permit the observations of sub-diurnal movements of suspended sediment, chlorophyll a concentration, floating derivations of green algae patches and evolutions of red tides. It is recognized that GOCI images provide also clearer views of sea fog, sea ice, yellow dust, wild fires, the effects of storms and even tsunamis than ever.

Since April, 2011, after the in-orbit-test of the satellite, the Korea Ocean Satellite Center (KOSC) of the Korea Institute of Ocean Science and Technology (KIOST), has taken charge of managing the GOCI data, implementing the reception, processing, $\mathrm{Cal} / \mathrm{Val}$ and their distribution to the users with a exclusive ownership. The GOCI data can be accessed through the KOSC's new website (http://kosc.kiost.ac) for non-commercial scientific and educational purposes. The GOCI Data Processing System (GDPS), developed by the KOSC, is software dedicated to perform the atmospheric correction and to generate the GOCI products including the images of the water color properties.

This special edition dedicated to the GOCI includes 13 articles which report the results of studies on overviews of the GOCI and the GDPS, sensitivity of the GOCI, comparison

E-mail: jhryu@kiost.ac,jishizak@hyarc.nagoya-u-ac.jp with other ocean color sensors, atmospheric correction, validation, and applications of GOCI data carried out by scientists from various countries. Lastly, we would like to thank OSJ for generously accepting the issue of this special edition as well as the Ministry of Land, Transport and Maritime Affairs for all the support in development and utilization of the GOCI. We sincerely hope that this special edition may foster further scientific researches and proper applications of new observation tools.

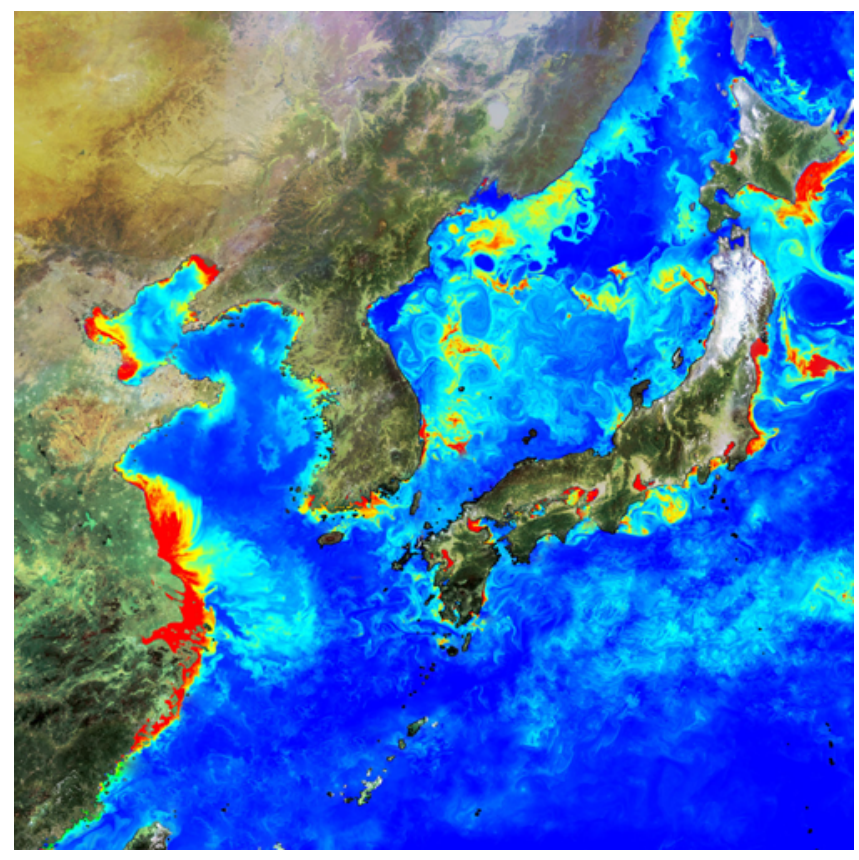

Fig. 1. Structure of chlorophyll distribution in the Northeast Asian Seas 